\title{
Ant1 mutant mice bridge the mitochondrial and serotonergic dysfunctions in bipolar disorder
}

\author{
Tomoaki M. Kato ${ }^{1,4} \cdot$ Mie Kubota-Sakashita ${ }^{1} \cdot$ Noriko Fujimori-Tonou $^{1} \cdot$ Fumihito Saitow $^{2} \cdot$ Satoshi Fuke $^{1} \cdot$ \\ Akira Masuda ${ }^{3} \cdot$ Shigeyoshi Itohara $^{3} \cdot$ Hidenori Suzuki $^{2} \cdot{\text { Tadafumi Kato } \mathbb{I}^{1}}^{1}$
}

Received: 16 January 2018 / Revised: 26 February 2018 / Accepted: 26 March 2018 / Published online: 11 June 2018

(c) The Author(s) 2018. This article is published with open access

\begin{abstract}
Although mitochondrial and serotonergic dysfunctions have been implicated in the etiology of bipolar disorder (BD), the relationship between these unrelated pathways has not been elucidated. A family of BD and chronic progressive external ophthalmoplegia (CPEO) caused by a mutation of the mitochondrial adenine nucleotide translocator 1 (ANT1, SLC25A4) implicated that ANT1 mutations confer a risk of BD. Here, we sequenced ANT1 in 324 probands of NIMH bipolar disorder pedigrees and identified two BD patients carrying heterozygous loss-of-function mutations. Behavioral analysis of brain specific Antl heterozygous conditional knockout (cKO) mice using lntelliCage showed a selective diminution in delay discounting. Delay discounting is the choice of smaller but immediate reward than larger but delayed reward and an index of impulsivity. Diminution of delay discounting suggests an increase in serotonergic activity. This finding was replicated by a 5-choice serial reaction time test. An anatomical screen showed accumulation of COX (cytochrome c oxidase) negative cells in dorsal raphe. Dorsal raphe neurons in the heterozygous cKO showed hyperexcitability, along with enhanced serotonin turnover in the nucleus accumbens and upregulation of Maob in dorsal raphe. These findings altogether suggest that mitochondrial dysfunction as the genetic risk of $\mathrm{BD}$ may cause vulnerability to $\mathrm{BD}$ by altering serotonergic neurotransmission.
\end{abstract}

These authors contributed equally: Tomoaki M. Kato, Mie KubotaSakashita, and Noriko Fujimori-Tonou

Electronic supplementary material The online version of this article (https://doi.org/10.1038/s41380-018-0074-9) contains supplementary material, which is available to authorized users.

$\triangle$ Tadafumi Kato

kato@brain.riken.jp

1 Laboratory for Molecular Dynamics of Mental Disorders, RIKEN Center for Brain Science, Wako, Saitama, Japan

2 Department of Pharmacology, Nippon Medical School, Tokyo, Japan

3 Laboratory for Behavioral Genetics, RIKEN Center for Brain Science, Wako, Saitama, Japan

4 Present address: Department of Fundamental Cell Technology, Center for iPS Cell Research and Application, Kyoto University, Kyoto, Japan

\section{Introduction}

Bipolar disorder is a major mental disorder characterized by mania and depression. Dysregulation in both monoaminergic systems [1] and mitochondrial calcium signaling [2] have been proposed in the etiology of bipolar disorder. However, the relationship between these apparently unrelated metabolic signaling systems has not been elucidated [3]. Clinical studies showed that around $20 \%$ of patients with mitochondrial disease have comorbid bipolar disorder [4-6], whereas $0.38 \%$ of patients with bipolar disorder had mutations of $P O L G$ (polymerase $\gamma$ ) causative for mitochondrial disease [7]. In a family of bipolar disorder and autosomal dominantly inherited chronic external ophthalmoplegia (CPEO), the L98P mutation of ANT1 (adenine nucleotide translocator 1, SLC25A4) was identified $[8,9]$. These findings suggest that central nervous system involvement in mitochondrial disease caused by ANT1 mutations confers a risk of bipolar disorder. ANT1 is named for its function as a translocator of adenosine triphosphate and adenosine diphosphate across the mitochondrial inner membrane. However, its modulatory role 
in the mitochondrial permeability transition pore (mPTP) has also drawn attention [10-12]. The mPTP plays a role in regulated cell death, and transient opening of the $\mathrm{mPTP}$ also regulates mitochondrial calcium signaling [13], which is consistent with the well-known calcium dysregulation hypothesis of bipolar disorder [14].

In this study, we searched for ANT1 mutations in patients with bipolar disorder, and identified two independent loss of function (LOF) mutations of ANTl. We investigated the relationship between heterozygous loss of function of ANT1 and bipolar disorder by generating a brain specific Antl conditional knockout (cKO) mouse. By behavioral screening, we identified that the heterozygous mice showed diminished delay discounting, that is the choice of smaller but immediate reward than larger but delayed reward and an index of impulsivity [15]. Consistent with this finding, the mice had enhanced serotoninergic activity. These findings together shed new light on the mechanism of how ANTl mutations may confer a risk for $\mathrm{BD}$.

\section{Materials and methods}

The study was approved by the Wako first ethics committee of RIKEN. All animal care and experimental procedure were in accordance with the guidelines for proper conduct of animal experiments published by Science Council of Japan and approved by RIKEN Wako Animal Experiment Committee. Methods and materials are described in detail in Supplemental Experimental Procedures.

\section{Subjects}

All four exons of the SLC25A4 gene were sequenced by PCR-direct sequencing in 324 probands of NIMH Genetics Initiative bipolar disorder pedigrees. Their diagnosis was bipolar I $(n=304)$, bipolar II disorder $(n=17)$, or schizoaffective disorder, bipolar type $(n=3)$.

\section{Animals}

Floxed exon 2-3 of Slc25a4 mouse line $\left(S l c 25 a 4^{\mathrm{tmla(EUCOMM)Wtsi}}\right)$ was obtained from International Knockout Mice Consortium (IKMC). Flp-transgenic mouse line (B6 ${ }^{\mathrm{Tg}(\mathrm{cat}-\mathrm{Flpe}) 36}$ ) was previously generated [16]. Nestin-Cre transgenic mouse line (B6.Cg ${ }^{\operatorname{Tg}(\text { Nes-cre) }}$ ${ }_{1 \mathrm{KIn} / \mathrm{J}}$ ) was obtained from Jackson laboratory. Using these mice lines, heterozygous and homozygous cKO mice of

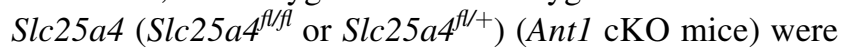
generated.

\section{Staining}

Staining methods and antibodies are described in detail in Supplementary Methods.

Fluorescent in situ hybridization was conducted as previously described [17]. Images were captured by a confocal microscope IX81 with FV1000 (Olympus Corporation, Tokyo, Japan), Observer Z1 with AxioVision 4.6 (Zeiss, Oberkochen, Germaney) or Nano Zoomer Digital Pathology system (Nanozoomer 2.0RS, Hamamatsu Photonics, Hamamatsu, Japan).

\section{Mouse behavioral screening with IntelliCage}

The IntelliCage apparatuses (NewBehavior AG, Zurich, Switzerland) were used for behavioral screening as described previously $[18,19]$. Male mice including 8 heterozygous cKO mice (Slc25a $4^{\mathrm{fl} / \mathrm{+}}$; Nes-Cre+), 10 homozygous cKO mice (Slc25a4 $4^{f / f}$; Nes-Cre+) and 6 controls $\left(\right.$ Slc $25 a 4^{f /+}$ or Slc25a4t/flf; Nes-Cre-), which were 20-27 week old, were used.

\section{5-choice serial reaction time task (5-CSRTT)}

The 5-CSRTT operant chamber (O'HARA \& Co., Tokyo, Japan) was used as previously described with minor modification [20]. Male mice including 8 controls $\left(S l c 25 a 4^{A l++}\right.$ or Slc25a4 $4^{f / f}$; Nes-Cre-), 8 heterozygous cKO mice $\left(\right.$ Slc $25 a 4^{f / /+}$; Nes-Cre+) and 8 homozygous cKO mice (Slc25a4 $4^{\text {flff }}$; Nes-Cre+), which were 8-13 week old at the beginning of training, were used for the analysis.

\section{Quantification of mtDNA deletion and mtDNA copy number}

Partially deleted mitochondrial DNA ( $\triangle$ mtDNA) and copy number of mtDNA was measured by quantitative PCR methods using SYBR Premix Ex Taq Kit (Takara Bio, Otsu, Japan) and QuantStudio $12 \mathrm{~K}$ Flex (Thermo Fisher Scientific, Waltham, MA) as described [21]. For quantification of mtDNA deletion 30-39 week old male mice were used. For mtDNA copy number analysis, 54-56 week old male mice were used. Control mice were Slc $25 a 4^{\mathrm{fl} /+}$ or

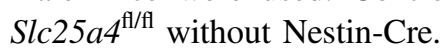

\section{Quantification of Ant1/Ant2 mRNAs}

mRNA expression of Ant1 (Slc25a4) and Ant2 (Slc25a5) were measured by quantitative PCR methods using SYBR Premix Ex Taq Kit (Takara Bio, Kusatsu, Japan) and QuantStudio $12 \mathrm{~K}$ Flex (Thermo Fisher Scientific, Waltham, MA). For these analysis, female 78-114 week old mice were used ( $n=3$ for each group). 
A

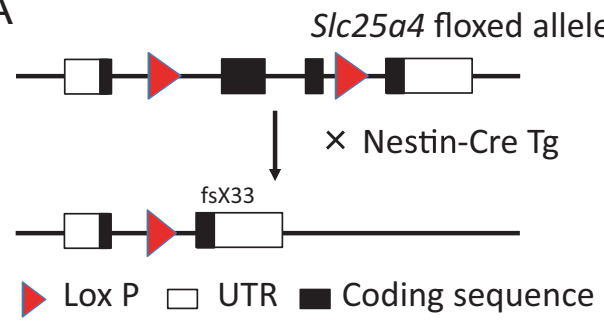

B
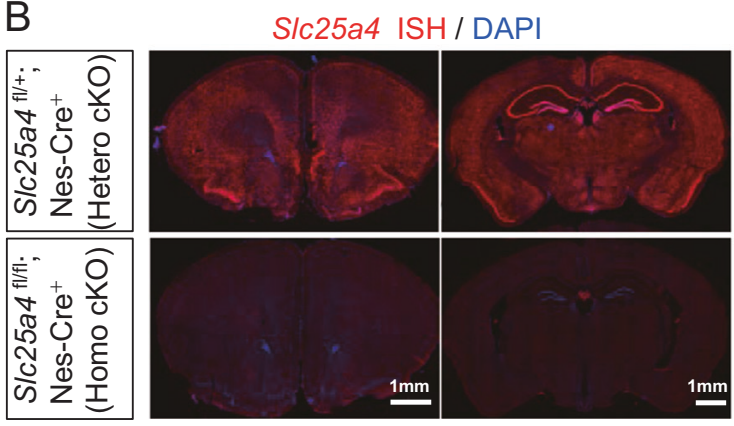

C

Frontal lobe
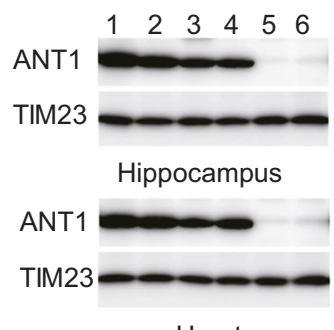

Heart

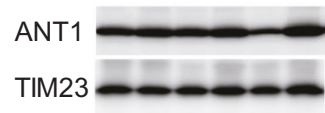

Muscle (thigh)

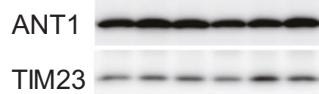

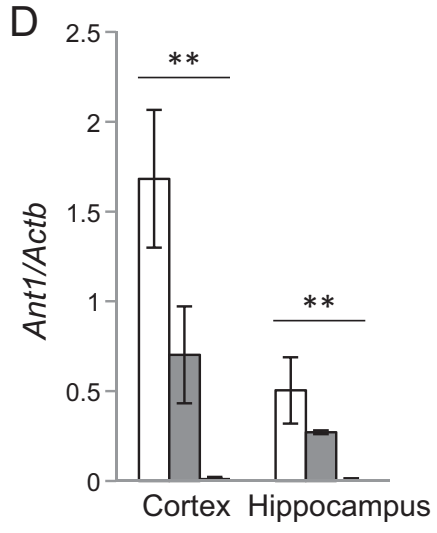

$\mathrm{F}$

Control

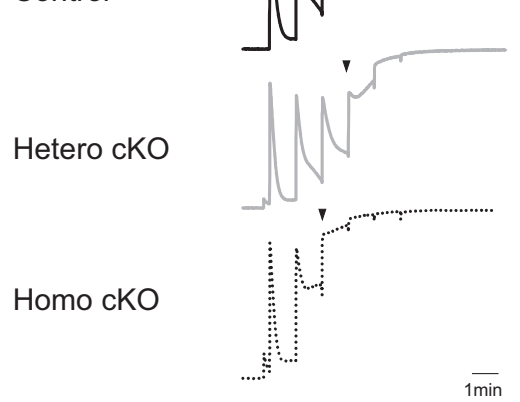

$\overline{1 \mathrm{~min}}$
E $\left.^{2.5}\right] \quad \square S / c 25 a 4^{\text {fl/fl }}$ (Control) $(\mathrm{N}=3)$

$\square$ Slc25a4 $4^{\mathrm{fl} /+} ;$ Nestin-Cre ${ }^{+}$(Hetero cKO) $(\mathrm{N}=3)$

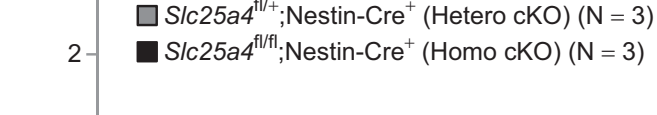

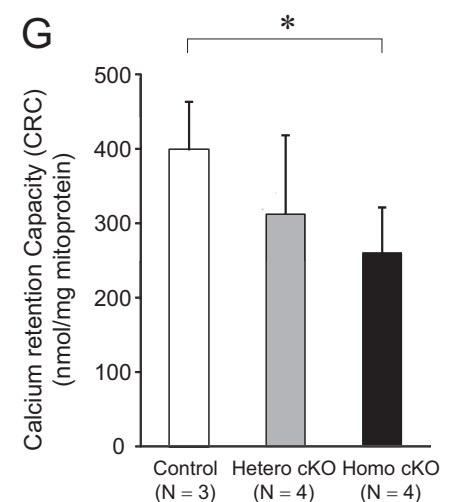

Fig. 1 Generation of brain-specific Ant1 conditional knockout (cKO) mice. a Structures of floxed Ant1 (Slc25a4) allele and conditionally knocked out (cKO) allele of Ant1. b In situ hybridization for Antl (red) and DAPI (blue) staining in the brains of heterozygous and homozygous Antl cKO mice. No mRNA expression was detected in homozygous cKO mice. c Western blotting of ANT1 and TIM23 (internal control for inner mitochondrial membrane) of brain tissues and muscle in heart or thigh from male $(1,3,5)$ and female $(2,4,6)$ control mice $(1,2)$ and heterozygous $(3,4)$ and homozygous $(5,6)$ Antl cKO mice. d, e Quantitative PCR analysis for Antl (d) and Ant2 (Slc25a5) (e) in the brains of control mice and heterozygous and homozygous Antl cKO mice. **P $<$ 0.005 by one way ANOVA. Post hoc test indicates significant difference of $A N T 1$ between all three groups for cortex and a significant difference between homozygous cKO and controls for hippocampus. No significant group difference was found for ANT2. $\mathbf{f}$ Representative charts of the experiments to measure calcium retention capacity (CRC). Vertical axis indicates Calcium green fluorescence intensity that reflects $\mathrm{Ca}^{2+}$ concentration of extra-mitochondrial fluid. Decay phase of each rise of extra-mitochondrial $\mathrm{Ca}^{2+}$ concentration reflects the uptake of $\mathrm{Ca}^{2+}$ by mitochondria. Arrow heads indicate the timing of opening of mitochondrial permeability transition pore (mPTP). Mitochondria from homozygous Antl cKO mice showed earlier mPTP opening, indicating smaller CRC. $\mathbf{g}$ Effect of loss of Antl on the mPTP opening. The vertical axis indicates the CRC. The data represents mean \pm SEM. $* P<0.05$ 


\section{Measurement of calcium retention capacity}

Brain mitochondria were isolated using a discontinuous Percoll gradient developed by Sims [22] with minor modifications [23]. Extra-mitochondrial free $\mathrm{Ca}^{2+}$ concentration $\left(\left[\mathrm{Ca}^{2+}\right]_{\mathrm{exm}}\right)$ was monitored with $200 \mathrm{nM}$ Calcium Green-5N (Thermo Fisher Scientific, Waltham, MA) (Ex $480 \mathrm{~nm}$, Em $540 \mathrm{~nm}$ ) in a 96 well plate at $30^{\circ} \mathrm{C}$ in a Drug Screening System (FDSS 3000, Hamamatsu Photonics, Hamamatsu, Japan). To evaluate the CRC, $10 \mu$ of $\mathrm{Ca}^{2+}$ solution was repeatedly added at 1-min intervals. Heterozygous cKO mice (Slc25a4 $4^{f /+}$; Nes-Cre +$)(n=4)$, homozygous cKO mice (Slc25a4 ${ }^{f / f}$; Nes-Cre+) $(n=4)$, and control mice $\left(\mathrm{Slc} 25 \mathrm{a} 4^{f / f}\right.$; Nes-Cre- $)(n=3)$ aged $8-27$ weeks were used for this analysis.

\section{Quantification of monoamine in tissue by HPLC}

Dopamine, noradrenaline and serotonin and their metabolites were measured by HPLC with an EICOMPAK SC-5ODS with electrochemical detector ECD-300 (Eicom Corporation, Kyoto, Japan). In this experiment, control mice included Slc25a4 ${ }^{+/+}$; Nestin-Cre+ and Slc $25 a 4^{+/+}$, Slc $25 a 4^{\mathrm{fl} /+}$ or Slc $25 a 4^{\mathrm{fl} / \mathrm{fl}}$ without Nestin-Cre. For this experiment, 88-103 week old male mice were used.

\section{Electrophysiological analysis}

Brain slices for experiments were prepared from 10-12week-old, male mice as described previously [24]. Whole cell patch-clamp recordings were acquired and controlled using the Axon 700B Multiclamp amplifer (Molecular Devices, CA, US) and pClamp11 acquisition software (Molecular Devices, CA, US). For this analysis, heterozygous Ant 1 cKO mice $\left(S l c 25 a 4^{f / /}\right.$; Nes-Cre +$)(n=4)$ and control mice $\left(\operatorname{Slc} 25 a 4^{f / /+}\right.$ without Nes-Cre) $(n=3)$ aged 8-12 week old were used.

\section{Statistical analysis}

Data were analyzed by Prism 4 (Graphpad softoware Inc., San Diego, CA), IBM SPSS Statistics 20 (IBM Japan, Tokyo, Japan), "R" (https://www.r-project.org/) or Kyplot (Kyence, Tokyo, Japan). For genetic association analysis, Fisher's exact probability test was used. In the comparison between the heterozygous or homozygous cKO mice and control mice, Student $t$-test was used. For place learning task and delay discounting task in IntelliCage, repeated measures ANOVA with Bonferroni's post hoc test was used with main effects of genotype and delay or day.

\section{Results}

\section{Identification of loss of function mutations in bipolar patients}

We sequenced the ANT1 gene in 324 NIMH probands with bipolar disorder and identified two patients carrying LOF mutations. One patient had a stop codon mutation p.Q85X, a substitution of $\mathrm{C}$ to $\mathrm{T}$ at $\mathrm{Chr} 4: 185,144,905$ [hg38] while the other had p.Q175RfsX38, a single nucleotide deletion at Chr 4: 185,145,174, causing a frameshift and premature stop codon (Supplementary Figures 1A-B), which was reported in a pedigree of recessive cardiomyopathy with comorbid depression and anxiety [25]. The frequency of LOF mutations (gain of stop codon or frame shift) for ANTI in BD $(2 / 324,0.61 \%)$ was significantly higher than that in the exome and genome data in the gnomAD database (10 of 128,632 [2 stop codons and 8 frameshift mutations], $0.000069 \%$, Fisher's exact probability test, $P=0.00040$, odds ratio $=79.7$ [95\%CI: 8.4-374.4]). Both mutations were on exon 3 and were thus predicted to undergo nonsense-mediated mRNA decay. Even when cDNAs of the predicted mutant mRNA encoding truncated proteins were constructed, protein expression of these mutants was markedly reduced in Neuro 2A cells (Supplementary Figure 1C). Although the two mutations did not show complete cosegregation with $\mathrm{BD}$ in the two pedigrees partly because of bilinear transmission (Supplementary Figure 1D), the significant association with high odds ratio suggested the role of these LOF mutations as a genetic risk factor for BD.

\section{Generation of brain-specific Ant1 cKO mice}

ANT1 mutations are known to cause a neuromuscular disorder, CPEO, and thus behavioral analyses must be performed using brain-specific mutant mice. We crossed floxed

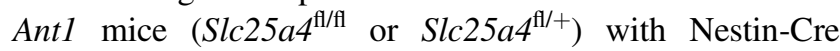
transgenic mice to generate a brain-specific cKO Antl mouse (Fig. 1a). We verified that the homozygous Antl cKO mice (Slc25a4 $4^{\mathrm{Al} / \mathrm{fl}}$; Nestin-Cre+) have no Ant1 mRNA (Fig. 1b) and protein (Fig. 1c) expression in the brain, and heterozygous Antl cKO mice (Slc25a4 ${ }^{\mathrm{f} / /+}$; Nestin-Cre+) have reduced Antl mRNA (Fig. 1d) compared to control mice $\left(\right.$ Slc $25 a 4^{+/+}$; Nestin-Cre+, Slc $25 a 4^{\mathrm{fl} /+}$ or Slc $25 a 4^{\mathrm{fl} / \mathrm{fl}}$; without Nestin-Cre). No compensatory upregulation of Ant2 mRNA was observed (Fig. 1e).

To examine the functional consequences of the loss of ANT1, we isolated mitochondria from Antl cKO mice. The mitochondrial calcium retention capacity was significantly lower in homozygous Antl cKO mice $(P=0.02)$ (Figs. 1f, g) suggesting that the mitochondria of homozygous Antl cKO mice are vulnerable to 
the mPTP opening. The calcium retention capacity of heterozygous Antl cKO mice did not significantly differ from controls.
Because the identified patients with bipolar disorder were heterozygous for LOF mutations in ANT1, we performed the murine behavioral characterization in heterozygous Antl
A

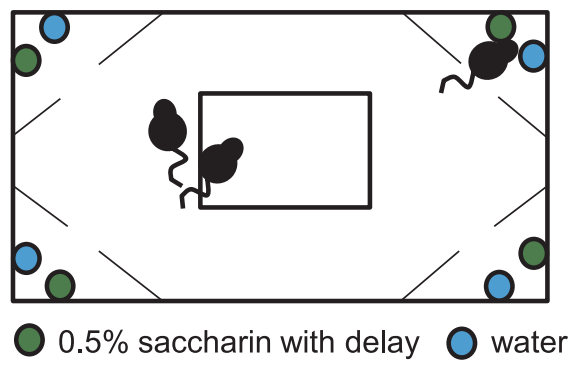

$B$

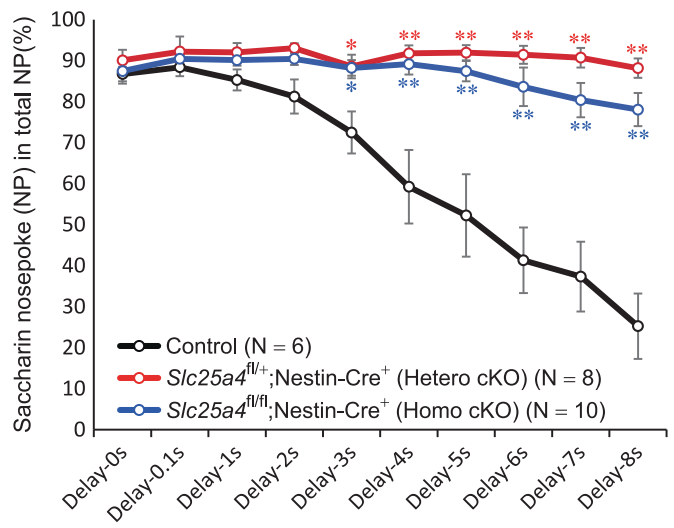

C
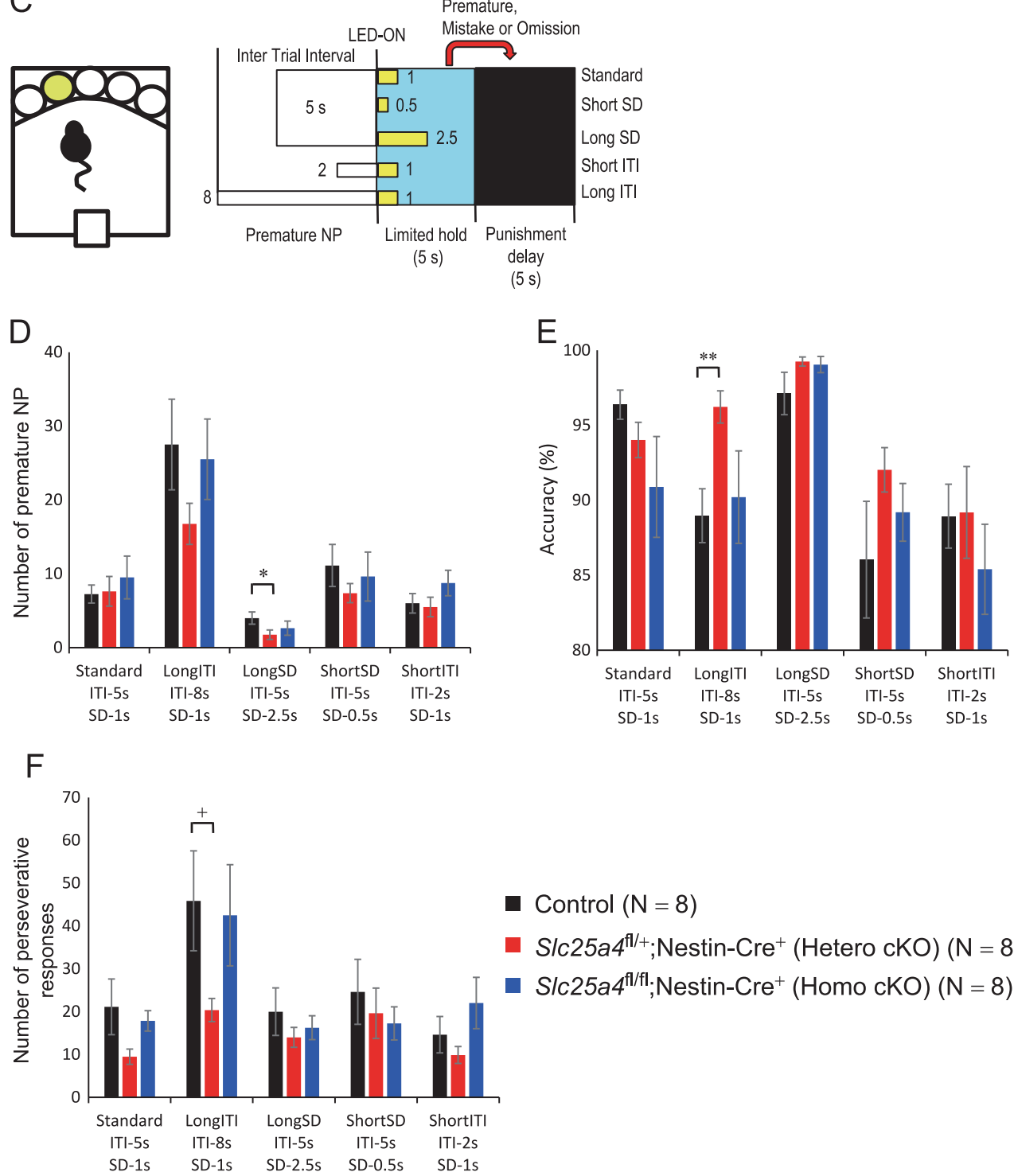

Control $(\mathrm{N}=8)$

- S/c25a $4^{\mathrm{fl} /+} ;$ Nestin-Cre ${ }^{+}$(Hetero cKO) $(\mathrm{N}=8)$

- Slc25a $4^{\mathrm{fl} / \mathrm{fl} \mid} ; \mathrm{Nestin}^{-\mathrm{Cre}^{+}}(\mathrm{Homo} \mathrm{cKO})(\mathrm{N}=8)$ 
Fig. 2 Behavioral analysis of Ant $1 \mathrm{cKO}$ mice by IntelliCage and fivechoice serial reaction time test (5-CSRTT). a Schematic drawing of delay discounting task in IntelliCage. The actions such as nose poking and licking of mice in each corner to drink running water (cyan) or $0.5 \%$ saccharin containing water (green) can be automatically recorded. b Percentages of nose poke counts for saccharin water in total nose poke counts in each day without or with activation delay after corner entry. Nose poke ratios for saccharin water of heterozygous ( $n$ $=8$, red) and homozygous ( $n=10$, blue) conditional knockout mice were higher than control mice ( $n=6$, black) in long delay ( $\geq 3 \mathrm{~s})$. Each circle represents mean and error bars indicate standard error of mean (SEM). $* * P<0.005 ; * P<0.05$ (Bonferroni's post hoc test after twoway repeated measures ANOVA). Red and blue asterisks indicate the comparisons of control vs heterozygous cKO, or control vs homozygous cKO, respectively. c Schematic drawing of 5-CSRTT. A sugar pellet was delivered to food magazine by a correct nose poke at an illuminated hole among five holes during limited hold. LED which was a sign for a correct hole was turned on after inter trial interval (ITI) for the defined period in each test. Premature or mistake nose poke and omission trial will lead to additional punishment delay in which room light was shut down for $5 \mathrm{~s}$. d Number of premature nose poke in heterozygous mutant mice was significantly decreased compared with that in control mice in long stimulus duration test. e Accuracy (percentage of correct nose poke / (correct nose poke + mistake)) was significantly higher in heterozygous Antl cKO mice compared with controls in long-ITI test. f Number of preservative responses was lower in heterozygous Ant $1 \mathrm{cKO}$ mice compared with controls in longITI test. ${ }^{*} P<0.005 ; * P<0.05,{ }^{+} P=0.05$. $S D$ stimulus duration, ITI inter-trial interval, $N P$ nose poke

cKO mice to model the human disorder. Homozygous cKO mice were examined as a reference control, and Slc25a $4^{\mathrm{H} /+}$ or Slc $25 a 4^{\mathrm{Il} / \mathrm{fl}}$ without Nestin-Cre, or Slc $25 a 4^{+/+}$; Nestin$\mathrm{Cre}+$ were also examined as controls.

\section{Behavioral phenotypes of brain-specific Ant1 cKO mice}

We performed behavioral screening using the IntelliCage (Fig. 2a). Indices for spatial learning, reverse learning, and attention did not differ between genotypes (Supplementary Figures 2A-E), but the heterozygous cKO mice showed a significantly decreased delay discounting (Fig. 2b). The preference for saccharin did not differ between genotypes $(F=1.0, \quad P=0.36$ by one way ANOVA) (Supplementary Figure 2F), suggesting that this finding is not due to altered reward value. Homozygous Antl cKO mice also showed a similar behavioral phenotype (Fig. 2b), although more similar to controls than heterozygous cKO mice.

To further characterize the behavioral phenotypes of heterozygous Antl cKO mice, we performed a 5-choice serial reaction time test (5-CSRTT), an established test to measure impulsivity [26] as a reflection of enhanced delay discounting (Figs. 2c-f, Supplementary Figures 2G-I). In this test, the number of premature nose pokes during long stimulus duration trials, which is an index of impulsivity, was significantly lower in heterozygous cKO mice than control mice (Fig. 2d). A decrease of impulsivity is equivalent to a decrease in delay discounting shown by the IntelliCage (Fig. 2b). The heterozygous cKO mice also showed better accuracy (Fig. 2e) and a lower number of perseverative responses than control mice in the long intertrial interval trials (Fig. 2f). Homozygous cKO mice did not show similar phenotypes in the 5-CSRTT for unknown reasons.

\section{Anatomical screen}

We searched for brain regions with mitochondrial dysfunction due to the heterozygous knockout of Antl by performing COX (cytochrome c oxidase)/SDH (succinate dehydrogenase) co-staining. COX is a mtDNA-encoded protein whereas SDH is a nuclear-encoded mitochondrial protein, and COX-negative cells were detected in a model mouse of mitochondrial disease [27]. In a sagittal section of aged homozygous Ant1 cKO mice, COX negative cells were detected preferentially in the dorsal raphe (DR) (Fig. 3a) and heterozygous Antl cKO mice also showed COX negative cells in the DR (Fig. 3b). Unexpectedly, however, a similar accumulation of COX negative cells was also detected in the DR of wild type mice (Fig. 3b). In this region, mtDNA deletions $(\triangle \mathrm{mtDNA})$ were not detectable both in cKO and control mice (Fig. 3c). Thus, the DR may have a selective vulnerability to mitochondrial dysfunction unrelated to the accumulation of $\triangle \mathrm{mtDNA}$, and the phenotype of the mutant mice might be caused by an interaction of the genotype and a general vulnerability of DR neurons to mitochondrial dysfunction. The copy number of mtDNA was significantly increased in DR of heterozygous cKO mice compared with control mice (Fig. 3d).

\section{Serotonergic dysfunction in Ant1 KO mice}

The activation of serotonergic neurons reportedly attenuates delay discounting [28], and therefore we examined serotonin turnover in the nucleus accumbens, which is innervated by DR serotonergic neurons and regulates impulsivity [29]. We found that serotonin turnover was enhanced in the nucleus accumbens of heterozygous KO mice (Fig. 3e). There was no significant difference of the serotonin turnover between NestinCre and wild type mice $(0.55 \pm 0.07$ vs $0.75 \pm 0.17$, respectively, $P=0.15, n=3$ for both groups). We also analyzed the gene expression level of Maob (monoamine oxidase B) that encodes a monoamine metabolizing enzyme on the mitochondrial outer membrane enriched in the DR [30]. To normalize expression levels of Maob by the number of serotonergic neurons within the sample, a serotonin neuron specific gene, Tph2 or Slc6a4, was used for a reference. We found that Maob mRNA was significantly increased in the DR 
A

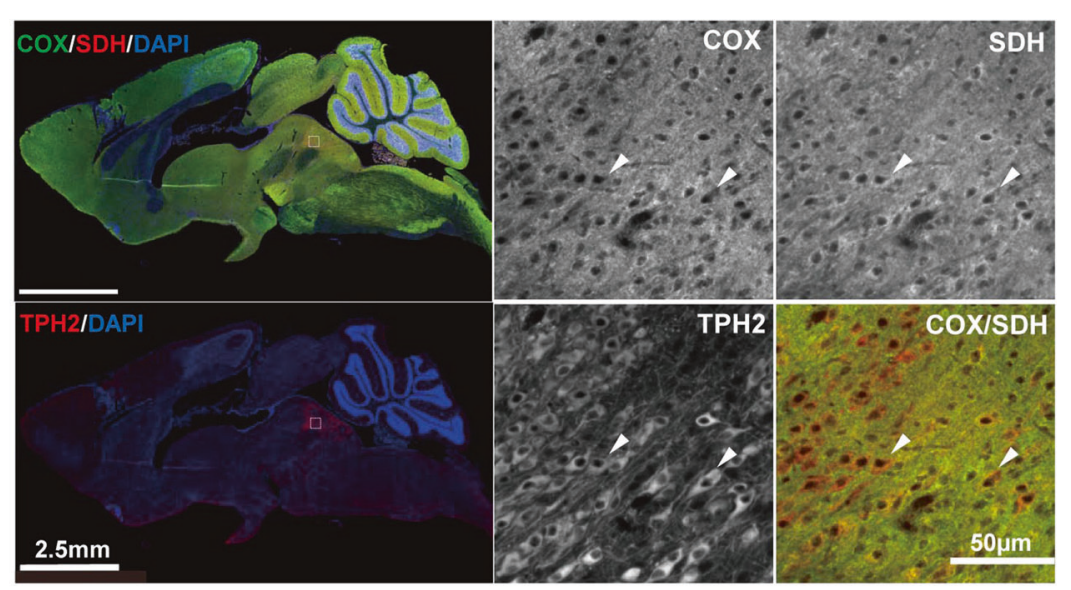

B
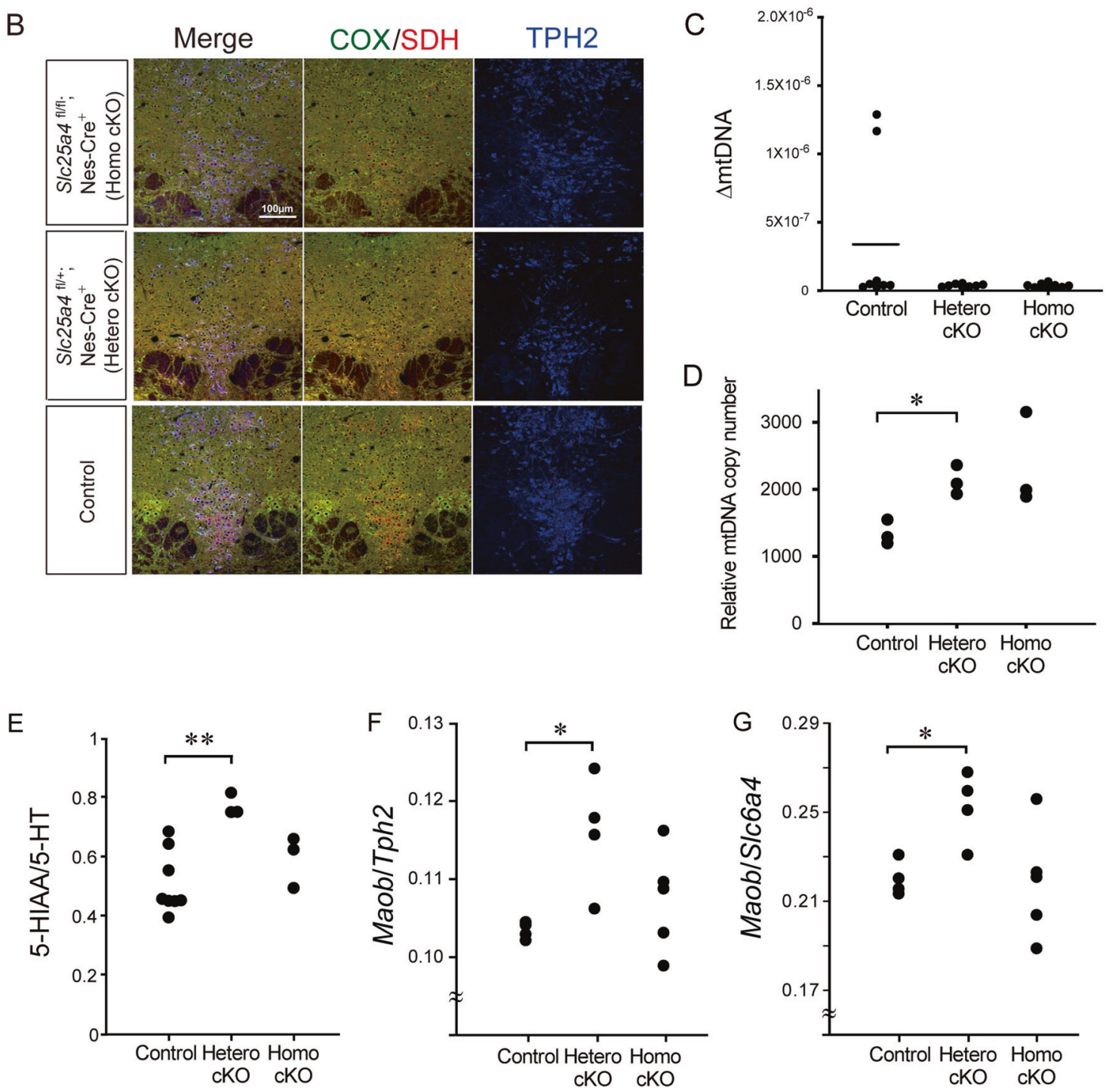

of heterozygous Antl cKO mice $(P<0.05)$ (Figs. 3f, g) consistent with the elevated turnover of serotonin in the nucleus accumbens.
To test whether serotonergic neurons were activated in the Antl cKO mice, we performed electrophysiological recordings from midbrain slices containing the DR. As 
Fig. 3 Anatomical screen by COX/SDH immunostaining of the brain suggested the role of dorsal raphe serotonergic neurons. a COX/SDH staining of a sagittal section of a 34 week old male homozygous Ant 1 cKO mouse. COX negative cells are seen as cells with red cytosol (positive signal of SDH [red] and no signal of COX [green]). Images were obtained by NDP and Axiovision. b COX/SDH staining of homozygous Ant $1 \mathrm{cKO}$ mouse (upper), heterozygous Ant $1 \mathrm{cKO}$ mouse (middle) and control mouse (bottom) (13 week old). Triple staining images for COX (green), SDH (red) and TPH2 (blue) indicate that COX negative cells are present in TPH2 positive serotonergic neurons. Images were obtained by confocal microscopy (FV1000). c Quantification of mtDNA deletions ( $\Delta$ mtDNAs) by quantitative PCR. The bar indicates average. There was no significant difference of $\Delta$ mtDNAs between heterozygous Antl cKO mice $(n=8)$ and control mice $(n=8)$. The homozygous cKO mice $(n=10)$ also did not accumulate mtDNA deletions. d Quantification of mtDNA copy number. A nuclear gene RNaseP was used as a reference. Heterozygous Ant1 cKO mice $(n=3)$ had significantly higher levels of mtDNA than control mice $(n=3)(P<0.01)$. The homozygous cKO mice $(n=3)$ also showed a tendency of elevation of mtDNA levels $(P=0.07)$. e The 5HIAA (5-hydroxyindole acetic acid)/5-HT (5-hydroxytryptamine, serotonin) ratio indicating turnover of serotonin is accelerated in heterozygous Ant1 cKO mice compared with control mice. $* * P<0.005$. Homozygous Ant1 cKO did not significantly differ from controls $(P=0.42)$. f, $\mathbf{g}$ Maob mRNA levels in dorsal raphe of Ant1 KO mice. Heterozygous Ant1 cKO mice showed significantly increased Maob/ Tph2 (f) and Maob/Slc6a4 (g) ratios compared with control mice

shown in Supplementary Table 1, both basic membrane properties and action potential properties of serotonergic neurons at the midline of the DR did not show significant differences between genotypes. However, the input-output relationship curves, which show the generation of action potentials by current injection was steeper in heterozygous Antl cKO mice than that of controls (Fig. 4) indicating that serotonergic neurons of heterozygous Antl cKO mice are more excitable.

\section{Discussion}

In this study, we identified two patients with bipolar disorder carrying loss of function mutations in ANT1 (Supplementary Figure 1). Brain-specific heterozygous cKO mice of Antl showed diminished delay discounting or reduced impulsivity by two behavioral tests (Fig. 2). Enhanced serotonin turnover (Fig. 3e) and hyperactivity of serotonergic neurons (Fig. 4) were consistent with the behavioral phenotype [28]. Increased mtDNA copy number in DR (Fig. 3d) might reflect an increased energy demand in the DR, and upregulation of Maob mRNA is considered a compensatory upregulation associated with enhanced serotonergic activity. These findings, together, suggest that the heterozygous loss of Antl in the brain causes a hyperserotonergic state and associated behavioral phenotypes.

The mechanism of how the heterozygous loss of function of Antl causes serotonergic hyperactivity is not known. Elevated intracellular calcium associated with
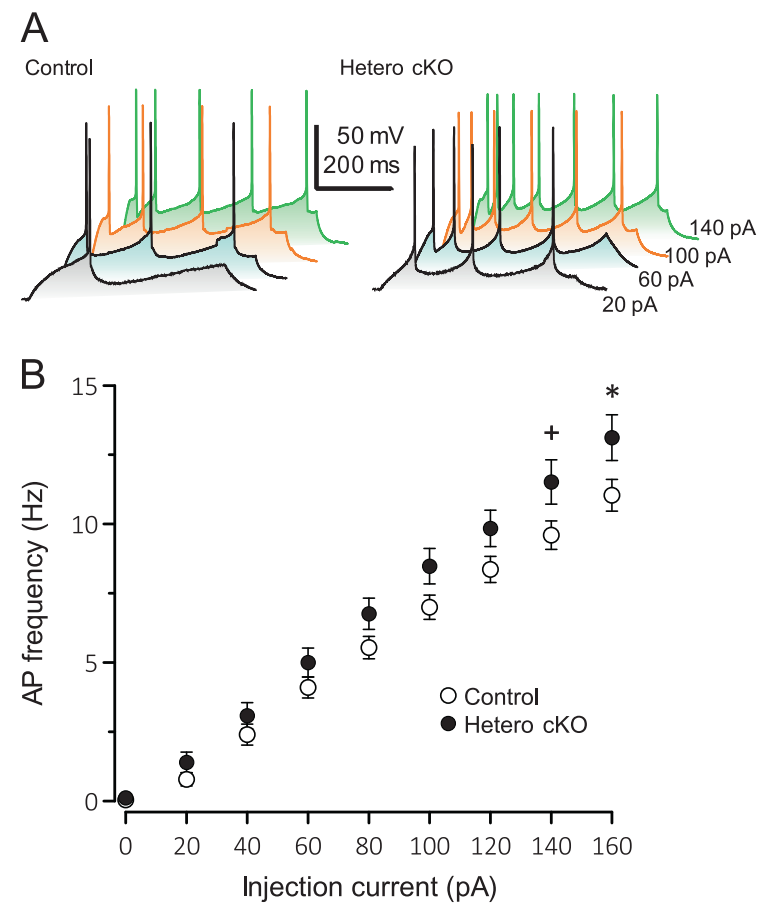

Fig. 4 DR serotonergic neurons in heterozygous Ant1 cKO mice show increased excitability. a Representative action potentials (APs) of DR neurons of the control and heterozygous Ant1 cKO mice that were generated by injecting currents of $20 \mathrm{pA}$ (black), $60 \mathrm{pA}$ (blue), $100 \mathrm{pA}$ (orange), and $140 \mathrm{pA}$ (green), respectively. b Pooled data of inputoutput relationship curves of DR serotonergic neurons in the control (open circles, $n=43$ ) and Ant 1 cKO (filled circles, $n=44$ ). APs were generated by injecting current steps from $0-160 \mathrm{pA}$ in increments of $20 \mathrm{pA}$ for $500 \mathrm{~ms}$. The frequency of AP generation were plotted. ${ }^{+} P=0.05 ; * P<0.05$ by Student $t$-test

depolarization is sequestered by mitochondria. The accumulation of intramitochondrial calcium results in a transient opening of the mPTP [31]. Ant1 reportedly has a modulatory effect on the mitochondrial permeability transition pore (mPTP) [10]. An altered mPTP function associated with heterozygous loss of Antl might affect the excitability of DR neurons.

A previous study reported exaggerated corticosterone responses to stress in homozygous conventional Antl KO mice [32]. Enhanced serotonergic function might also underlie this finding, because serotoninergic stimulation is known to activate the hypothalamic-pituitary-adrenal axis [33].

Why are serotonergic neurons preferentially affected by the heterozygous loss of Ant1? Monoamines are metabolized by monoamine oxidases (MAOs) on the mitochondrial outer membrane. Metabolism of monoamines by MAOs is accompanied by the generation of hydrogen peroxide, a reactive oxygen species [30]. Enhanced serotonin release by 3,4-methylenedioxymethamphetamine (MDMA) can decrease COX protein levels, which can be rescued by an MAO-B inhibitor [34]. 
We speculate that serotonergic neurons may be intrinsically vulnerable to mitochondrial dysfunction, and the hyperserotonergic state may further impair mitochondrial function in a vicious cycle. The results of the COX/SDH staining in this study support this hypothesis. The degeneration of DR serotonergic neurons is frequently seen in Parkinson's disease, in which mitochondrial dysfunction is implicated, and this might at least partly explain the non-motor symptoms of this disease [35]. In Parkinson's disease, 8-hydroxyguanosine is accumulated in substantia nigra dopaminergic neurons, and this is also true for the DR [36]. Thus, the mitochondrial dysfunction phenotype due to the heterozygous cKO of Antl is predominantly seen in serotonergic neurons, which may have an intrinsic vulnerability to mitochondrial dysfunction. The mechanism of how COX immunoreactivity is reduced in the DR is not known because the present study did not show an increase of $\triangle$ mtDNA levels or a decrease of mtDNA copy number. Reduction of COX immunoreactivity in DR neurons might be regulated by other types of mtDNA abnormalities and/or at the protein level [37].

Mitochondrial dysfunction has been implicated in bipolar disorder based on several lines of evidence [2] including altered energy metabolism detected by magnetic resonance spectroscopy [38], comorbidity with mitochondrial diseases $[4,7]$, and findings in postmortem brains including an accumulation of $\Delta$ mtDNAs [39, 40], altered gene expression of mitochondria-related genes [41], altered morphology of mitochondria [42], and decreased activity of mitochondrial complex I [43]. On the other hand, serotonergic dysfunction in bipolar disorder has been implicated by evidence including the maniainducing effect of antidepressants that inhibits the serotonin transporter and thereby activates serotonin [44], the efficacy of atypical antipsychotics that inhibits serotonergic neurotransmission [45], altered mRNA expression levels of serotonergic receptors in postmortem brain [46], altered DNA methylation of serotonin transporter gene [47], altered serotonin transporter binding in the brain by positron emission tomography [48], and levels of cerebrospinal fluid metabolites, among others [14]. The present findings provide a potential missing link between these two lines of evidence. Because the two LOF mutations identified in this study were not cosegregated with bipolar disorder in the two pedigrees, they are not "causative" mutations. However, their significant association suggests that the heterozygous LOF mutations of ANT1 confer the risk of bipolar disorder.

There are several limitations in this study. Notably, it is unknown why homozygous Antl cKO mice do not show behavioral alterations in some experiments. However, such non-linear dynamics are inherent to complex biological systems such as the brain. Secondly, Nestin-Cre mice reportedly have some behavioral alterations [49], which could in principle confound the results. However, we verified that this transgene did not affect serotonin turnover and excitability of DR serotonergic neurons (Supplementary Figure 3). Thirdly, behavioral analysis is confounded by genetic background [50]. Although all the mice used were generated and/or kept under the background of C57BL/6, there are subtle behavioral differences even among C57BL/ 6 substrains [51]. We therefore used the mice after backcrossing with C57BL/6J, though the number of generations might not be enough to rule out a possible effect of substrains. Finally, the results in calcium retention capacity are not consistent with previous studies that showed a loss of Antl causing an increase in calcium retention capacity [52]. This discrepancy might be due to differences in experimental conditions or mouse strains.

In summary, our current findings suggest that mitochondrial dysfunction caused by heterozygous loss of Antl can cause alterations of serotonergic activity, a first step toward understanding the complex neurobiological processes underlying bipolar disorder subtypes.

Acknowledgements We are grateful to Ms. Mizue Kametani and Dr. Hirochika Kawakami for technical assistance. We thank Dr. Katsuhide Igarashi and Ms. Maky Otsuka (Hoshi University School of Pharmacy and Pharmaceutical Sciences) for advising us the method for the dissection of dorsal raphe. We also thank Research Resource Center and BSI-Olympus Collaboration Center, RIKEN Brain Science Institute, for technical assistance. The work was supported by JSPS KAKENHI Grant Number JP27860956 to T.M.K. and JP17H01573 to T.K. and the Advanced Genome Research and Bioinformatics Study to Facilitate Medical Innovation (GRIFIN) from the Japan Agency for Medical Research and Development (AMED) (16815678) to TK.

Author contributions TMK and TK conceived the study design. TMK and NFT performed the molecular, anatomical, and behavioral experiments. MKS performed the experiments on isolated mitochondria and a part of the immunohistochemical analyses. FS and HS performed the electrophysiological experiments. SF sequenced the Ant1 gene in human samples. AM and SI conceived the method for the IntelliCage analysis. TMK and TK wrote the paper.

\section{Compliance with ethical standards}

Conflict of interest The authors declare that they have no conflict of interest.

Open Access This article is licensed under a Creative Commons Attribution 4.0 International License, which permits use, sharing, adaptation, distribution and reproduction in any medium or format, as long as you give appropriate credit to the original author(s) and the source, provide a link to the Creative Commons license, and indicate if changes were made. The images or other third party material in this article are included in the article's Creative Commons license, unless indicated otherwise in a credit line to the material. If material is not included in the article's Creative Commons license and your intended use is not permitted by statutory regulation or exceeds the permitted use, you will need to obtain permission directly from the copyright holder. To view a copy of this license, visit http://creativecommons. org/licenses/by/4.0/. 


\section{References}

1. Goodwin FK, Jamison KR. Manic-depressive illness. Bipolar disorders and recurrent depression. 2nd Edition. New York, NY: Oxford University Press; 2007.

2. Kato T, Kato N. Mitochondrial dysfunction in bipolar disorder. Bipolar Disord. 2000;2:180-90.

3. Manji H, Kato T, Di Prospero NA, Ness S, Beal MF, Krams M, et al. Impaired mitochondrial function in psychiatric disorders. Nat Rev Neurosci. 2012;13:293-307.

4. Fattal O, Link J, Quinn K, Cohen BH, Franco K. Psychiatric comorbidity in 36 adults with mitochondrial cytopathies. CNS Spectr. 2007;12:429-38.

5. Mancuso M, Orsucci D, Ienco EC, Pini E, Choub A, Siciliano G. Psychiatric involvement in adult patients with mitochondrial disease. Neurol Sci. 2013;34:71-4.

6. Inczedy-Farkas G, Remenyi V, Gal A, Varga Z, Balla P, UdvardyMeszaros A, et al. Psychiatric symptoms of patients with primary mitochondrial DNA disorders. Behav Brain Funct. 2012;8:9.

7. Kasahara T, Ishiwata M, Kakiuchi C, Fuke S, Iwata N, Ozaki N, et al. Enrichment of deleterious variants of mitochondrial DNA polymerase gene (POLG1) in bipolar disorder. Psychiatry Clin Neurosci. 2017;71:518-29.

8. Siciliano G, Tessa A, Petrini S, Mancuso M, Bruno C, Grieco GS, et al. Autosomal dominant external ophthalmoplegia and bipolar affective disorder associated with a mutation in the ANT1 gene. Neuromuscul Disord. 2003;13:162-5.

9. El-Khoury R, Sainsard-Chanet A. Suppression of mitochondrial DNA instability of autosomal dominant forms of progressive external ophthalmoplegia-associated ANT1 mutations in Podospora anserina. Genetics. 2009;183:861-71.

10. Graham BH, Waymire KG, Cottrell B, Trounce IA, MacGregor GR, Wallace DC. A mouse model for mitochondrial myopathy and cardiomyopathy resulting from a deficiency in the heart/ muscle isoform of the adenine nucleotide translocator. Nat Genet. 1997;16:226-34.

11. Zamzami N, Kroemer G. The mitochondrion in apoptosis: how Pandora's box opens. Nat Rev Mol Cell Biol. 2001;2:67-71.

12. Bernardi P, Di Lisa F. The mitochondrial permeability transition pore: molecular nature and role as a target in cardioprotection. $\mathbf{J}$ Mol Cell Cardiol. 2015;78:100-6.

13. Agarwal A, Wu PH, Hughes EG, Fukaya M, Tischfield MA, Langseth AJ, et al. Transient opening of the mitochondrial permeability transition pore induces microdomain calcium transients in astrocyte processes. Neuron. 2017;93:587-605.

14. Sigitova E, Fisar Z, Hroudova J, Cikankova T, Raboch J. Biological hypotheses and biomarkers of bipolar disorder. Psychiatry Clin Neurosci. 2017;71:77-103.

15. Rachlin H, Green L. Commitment, choice and self-control. J Exp Anal Behav. 1972;17:15-22.

16. Kanki H, Suzuki H, Itohara S. High-efficiency CAG-FLPe deleter mice in C57BL/6J background. Exp Anim. 2006;55:137-41.

17. Shimojo H, Ohtsuka T, Kageyama R. Oscillations in notch signaling regulate maintenance of neural progenitors. Neuron. 2008;58:52-64.

18. Kobayashi Y, Sano Y, Vannoni E, Goto H, Suzuki H, Oba A, et al. Genetic dissection of medial habenula-interpeduncular nucleus pathway function in mice. Front Behav Neurosci. 2013;7:17.

19. Masuda A, Kobayashi Y, Kogo N, Saito T, Saido TC, Itohara S. Cognitive deficits in single App knock-in mouse models. Neurobiol Learn Mem. 2016;135:73-82.

20. Asinof SK, Paine TA. The 5-choice serial reaction time task: a task of attention and impulse control for rodents. J Vis Exp. 2014;90:e51574.
21. Kasahara T, Takata A, Kato TM, Kubota-Sakashita M, Sawada T, Kakita A, et al. Depression-like episodes in mice harboring mtDNA deletions in paraventricular thalamus. Mol Psychiatry. 2016;21:39-48.

22. Sims NR. Rapid isolation of metabolically active mitochondria from rat brain and subregions using Percoll density gradient centrifugation. J Neurochem. 1990;55:698-707.

23. Kubota M, Kasahara T, Nakamura T, Ishiwata M, Miyauchi T, Kato T. Abnormal $\mathrm{Ca}^{2+}$ dynamics in transgenic mice with neuron-specific mitochondrial DNA defects. J Neurosci. 2006;26: 12314-24.

24. Gocho Y, Sakai A, Yanagawa Y, Suzuki H, Saitow F. Electrophysiological and pharmacological properties of GABAergic cells in the dorsal raphe nucleus. J Physiol Sci. 2013;63:147-54.

25. Strauss KA, DuBiner L, Simon M, Zaragoza M, Sengupta PP, Li $\mathrm{P}$, et al. Severity of cardiomyopathy associated with adenine nucleotide translocator-1 deficiency correlates with mtDNA haplogroup. Proc Natl Acad Sci USA. 2013;110:3453-8.

26. Robinson ES, Eagle DM, Economidou D, Theobald DE, Mar AC, Murphy ER, et al. Behavioural characterisation of high impulsivity on the 5-choice serial reaction time task: specific deficits in "waiting" versus "stopping". Behav Brain Res. 2009;196:310-6.

27. Tyynismaa H, Mjosund KP, Wanrooij S, Lappalainen I, Ylikallio E, Jalanko A, et al. Mutant mitochondrial helicase Twinkle causes multiple mtDNA deletions and a late-onset mitochondrial disease in mice. Proc Natl Acad Sci USA. 2005;102:17687-92.

28. Miyazaki KW, Miyazaki K, Tanaka KF, Yamanaka A, Takahashi A, Tabuchi S, Doya K. Optogenetic activation of dorsal raphe serotonin neurons enhances patience for future rewards. Curr Biol. 2014;24:2033-40.

29. Winstanley CA, Theobald DE, Dalley JW, Robbins TW. Interactions between serotonin and dopamine in the control of impulsive choice in rats: therapeutic implications for impulse control disorders. Neuropsychopharmacology. 2005;30:669-82.

30. Bortolato M, Chen K, Shih JC. Monoamine oxidase inactivation: from pathophysiology to therapeutics. Adv Drug Deliv Rev. 2008;60:1527-33.

31. Nguyen KT, Barrett JN, Garcia-Chacon L, David G, Barrett EF. Repetitive nerve stimulation transiently opens the mitochondrial permeability transition pore in motor nerve terminals of symptomatic mutant SOD1 mice. Neurobiol Dis. 2011;42:381-90.

32. Picard M, McManus MJ, Gray JD, Nasca C, Moffat C, Kopinski $\mathrm{PK}$, et al. Mitochondrial functions modulate neuroendocrine, metabolic, inflammatory, and transcriptional responses to acute psychological stress. Proc Natl Acad Sci USA. 2015;112: E6614-23.

33. Heisler LK, Pronchuk N, Nonogaki K, Zhou L, Raber J, Tung L, et al. Serotonin activates the hypothalamic-pituitary-adrenal axis via serotonin 2C receptor stimulation. J Neurosci. 2007; $27: 6956-64$.

34. Alves E, Summavielle T, Alves CJ, Gomes-da-Silva J, Barata JC, Fernandes E, et al. Monoamine oxidase-B mediates ecstasyinduced neurotoxic effects to adolescent rat brain mitochondria. J Neurosci. 2007;27:10203-10.

35. Qamhawi Z, Towey D, Shah B, Pagano G, Seibyl J, Marek K, et al. Clinical correlates of raphe serotonergic dysfunction in early Parkinson's disease. Brain. 2015;138:2964-73.

36. Zhang J, Perry G, Smith MA, Robertson D, Olson SJ, Graham DG, et al. Parkinson's disease is associated with oxidative damage to cytoplasmic DNA and RNA in substantia nigra neurons. Am J Pathol. 1999;154:1423-9.

37. Huttemann M, Lee I, Grossman LI, Doan JW, Sanderson TH. Phosphorylation of mammalian cytochrome $\mathrm{c}$ and cytochrome $\mathrm{c}$ oxidase in the regulation of cell destiny: respiration, apoptosis, and human disease. Adv Exp Med Biol. 2012;748:237-64. 
38. Kato T, Takahashi S, Shioiri T, Inubushi T. Brain phosphorous metabolism in depressive disorders detected by phosphorus-31 magnetic resonance spectroscopy. J Affect Disord. 1992; 26:223-30.

39. Kato T, Stine OC, McMahon FJ, Crowe RR. Increased levels of a mitochondrial DNA deletion in the brain of patients with bipolar disorder. Biol Psychiatry. 1997;42:871-5.

40. Shao L, Martin MV, Watson SJ, Schatzberg A, Akil H, Myers $\mathrm{RM}$, et al. Mitochondrial involvement in psychiatric disorders. Ann Med. 2008;40:281-95.

41. Konradi C, Eaton M, MacDonald ML, Walsh J, Benes FM, Heckers S. Molecular evidence for mitochondrial dysfunction in bipolar disorder. Arch Gen Psychiatry. 2004;61:300-8.

42. Cataldo AM, McPhie DL, Lange NT, Punzell S, Elmiligy S, Ye $\mathrm{NZ}$, et al. Abnormalities in mitochondrial structure in cells from patients with bipolar disorder. Am J Pathol. 2010;177:575-85.

43. Andreazza AC, Shao L, Wang JF, Young LT. Mitochondrial complex I activity and oxidative damage to mitochondrial proteins in the prefrontal cortex of patients with bipolar disorder. Arch Gen Psychiatry. 2010;67:360-8.

44. Peet M. Induction of mania with selective serotonin re-uptake inhibitors and tricyclic antidepressants. $\mathrm{Br} \mathrm{J}$ Psychiatry. 1994;164:549-50.

45. Kanba S, Kato T, Terao T, Yamada K. Guideline for treatment of bipolar disorder by the Japanese Society of Mood Disorders, 2012. Psychiatry Clin Neurosci. 2013;67:285-300.
46. Lopez-Figueroa AL, Norton CS, Lopez-Figueroa MO, ArmelliniDodel D, Burke S, Akil H, et al. Serotonin 5-HT1A, 5-HT1B, and 5HT2A receptor mRNA expression in subjects with major depression, bipolar disorder, and schizophrenia. Biol Psychiatry. 2004;55:225-33.

47. Sugawara H, Iwamoto K, Bundo M, Ueda J, Miyauchi T, Komori A, et al. Hypermethylation of serotonin transporter gene in bipolar disorder detected by epigenome analysis of discordant monozygotic twins. Transl Psychiatry. 2011;1:e24.

48. Cannon DM, Ichise M, Rollis D, Klaver JM, Gandhi SK, Charney DS, et al. Elevated serotonin transporter binding in major depressive disorder assessed using positron emission tomography and [11C]DASB; comparison with bipolar disorder. Biol Psychiatry. 2007;62:870-7.

49. Giusti SA, Vercelli CA, Vogl AM, Kolarz AW, Pino NS, Deussing JM, et al. Behavioral phenotyping of Nestin-Cre mice: implications for genetic mouse models of psychiatric disorders. J Psychiatr Res. 2014;55:87-95.

50. Hiroi N. Critical reappraisal of mechanistic links of copy number variants to dimensional constructs of neuropsychiatric disorders in mouse models. Psychiatry Clin Neurosci. 2018;72:301-21.

51. Matsuo N, Takao K, Nakanishi K, Yamasaki N, Tanda K, Miyakawa T. Behavioral profiles of three C57BL/6 substrains. Front Behav Neurosci. 2010;4:29.

52. Lee J, Schriner SE, Wallace DC. Adenine nucleotide translocator 1 deficiency increases resistance of mouse brain and neurons to excitotoxic insults. Biochim Biophys Acta. 2009;1787:364-70. 